\title{
A XANES study of chromophores: the case of black glass
}

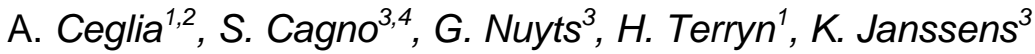 \\ 1 Dep. of Electrochemical and Surface Engineering, Vrije Universiteit Brussel, Pleinlaan 2, B-1050 Brussels, Belgium \\ 2 Dep. of Applied Physics and Photionics, Vrije Universiteit Brussel, Pleinlaan 2, B-1050 Brussels, Belgium \\ ${ }^{3}$ Dept. of Chemistry, University of Antwerp, Groenenborgerlaan 171, B-2610 Antwerp, Belgium \\ ${ }^{4}$ Dep. of Plant- and Environmental Sciences, Norwegian University of Life Sciences, PO Box 5003, 1432 Aas, Norway
}

This report summarizes the results published in a paper recently submitted to Analytical Chemistry [1]. At DORIS Beamline L we examined several Roman black glass fragments using Fe K-edge Xray absorption near edge (XANES). The selected archaeological glass samples cover the period 1st5 th century AD along ten different sites of the North Western provinces of the Roman Empire. The main goal was to determine the $\mathrm{Fe}^{3+} / \Sigma \mathrm{Fe}$, since this ratio influences glass-colour and can result in hues ranging from brown (in presence of sulphur) over green to blue or optionally even colourless glass. 27 Fragments from vessel glass and glass jewelry were selected from different periods in Roman history in which black glass was considered fashionable.

At DORIS Beamline L a Si(111) double crystal monochromator was used, having an energy resolution $\triangle \mathrm{E} / \mathrm{E}$ of about $10^{-4}$. A transmission XANES spectrum was recorded from a metallic Fe reference foil and used to provide an accurate energy calibration for all spectra; first inflection point of the Fe-K edge was set to $7112 \mathrm{eV}$. A slight detuning of the two monochromator crystals was used to remove high energy harmonics from the incident $\mathrm{X}$-ray beam. $\mathrm{FeCl}_{3}$ and $\mathrm{FeSO}_{4}$ reference compounds were recorded in transmission mode and provided reference spectra for ferrous (II) and ferric (III) iron. Self-absorption was limited by diluting these powdered compounds to app. 2 wt $\%$ with cellulose and pressing it into a pellet. In addition two glasses were recorded in fluorescence mode with a different total amount of $\mathrm{Fe}(0.1 \mathrm{wt} \%$ and $0.6 \mathrm{wt} \%)$ but an identical $\mathrm{Fe}^{3+} / \Sigma \mathrm{Fe}$ ratio (0.67). The fluorescence yield was recorded as a function of the incoming X-ray energy using a Vortex detector at an angle of $45^{\circ}$ with respect to the incoming beam, while also the sample surface is oriented at $45^{\circ}$ to the incoming beam. ROI fitting was performed around the Fe $\mathrm{K} \alpha$ line (6113$6692 \mathrm{eV})$. XANES spectra were collected form $\sim 32 \mathrm{eV}$ below to $\sim 130 \mathrm{eV}$ above the Fe-K edge using different energy intervals $(7090 \mathrm{eV}-7105 \mathrm{eV}: 0.25 \mathrm{eV}, 7105 \mathrm{eV}-7120 \mathrm{eV}: 0.1 \mathrm{eV}, 7120 \mathrm{eV}$ - $7250 \mathrm{eV}: 0.25 \mathrm{eV}$ ); a measuring time of $1 \mathrm{~s}$ was used for each energy step resulting in a $\sim 20 \mathrm{~min}$ measuring time per XANES spectrum. Three repeats were recorded for each Fe model compound in order to acquire virtually noise-free spectra. Measurements on real glass samples were performed under the same conditions, however the focused primary beam was made as large as possible, circa $780 \times 520 \mu \mathrm{m}^{2}$. For all XANES spectra, the normalisation was performed by means of the software package ATHENA. An edge-step normalisation was performed by a linear pre-edge subtraction and by regression of an (in general) third degree polynomial beyond the edge [2].

The pre-edge peak is extracted using an arctangent function to describe the background (Fig. 1). In literature different functions have been employed for the description of the pre-edge features [3]. When the same mathematical model is used throughout the entire data set, the conclusions will be consistent. Problems may arise, however, when comparing the data to literature values [4]. For the glass samples, the Fe-K pre-edge features have been fitted using two Voigt peaks with $2 \mathrm{eV}$ of average width [3-7]. For the reference compounds, it was necessary to include a third peak function to obtain good fits. All peaks were constrained to have a 50\% Lorentzian-50\% Gaussian shape. 
The centroid of the extracted pre-edge for an certain oxidation state always occurs at the same position irrespective to the coordination. Therefore its position can be used to determine the $\mathrm{Fe}^{3+} / \Sigma \mathrm{Fe}$, which is a relevant parameter testifying to the redox conditions during the production of coloured glass.

The results obtained suggest that in an earlier stage (first century AD) black glass was produced in a single step, under controlled reducing conditions $\left(\mathrm{Fe}^{3+} / \Sigma \mathrm{Fe} \approx\right.$ 0.17). After the second half of the $2^{\text {nd }}$ century $\mathrm{AD}$, instead, the $\mathrm{Fe}^{3+} / \Sigma \mathrm{Fe}$ values (0.4-0.5) are similar to what Arletti et al. [8] found for naturally coloured glass. This corroborates the hypothesis that large quantities of iron were added in a secondary step to turn naturally coloured glass into black. This was most likely achieved without requiring specific control of the redox conditions in the furnace.

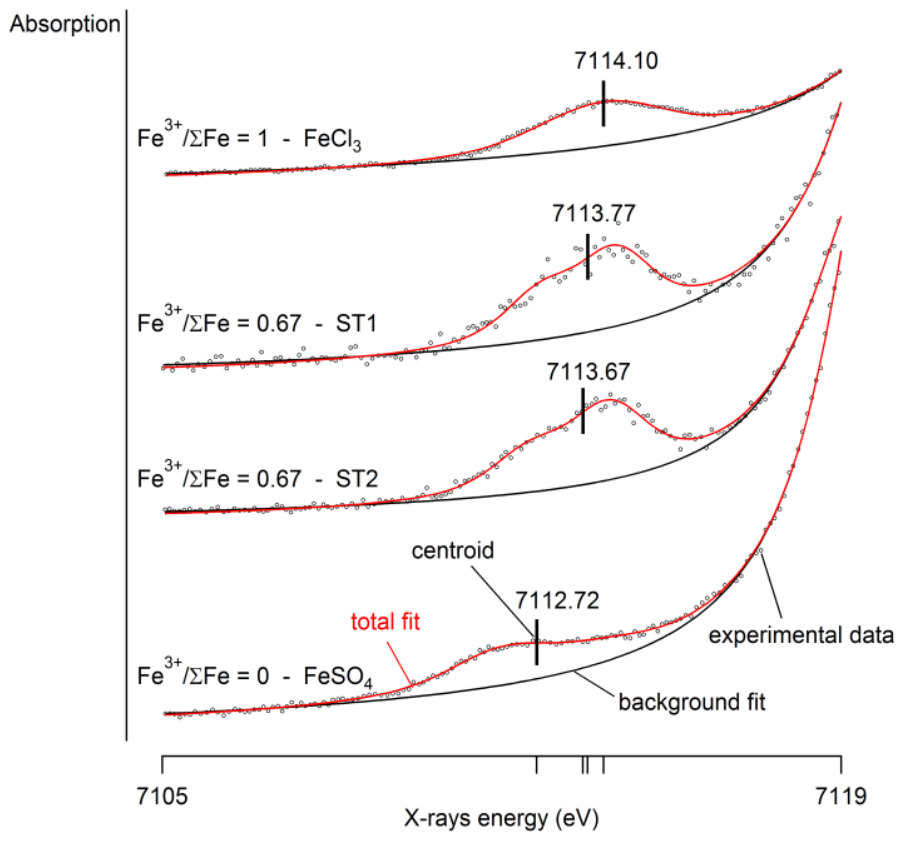

Figure 1: Pre-edge feature of reference glasses and referece compounds. The background is fitted using an acrctangent function, while the pre-edge region is described by two or three Voigt functions.

\section{References}

[1] A. Ceglia, et al., Analytical Chemistry, submitted (2013)

[2] B. Ravel, et al., Journal of Synchrotron Radiation, 12, 537-541(2005)

[3] F. Farges, et al., Journal of Non-Crystalline Solids, 344(3), 176-188(2004)

[4] A.J. Berry, et al., American Mineralogist, 88(7), 967-977(2003)

[5] A.J. Berry, et al., Nature, 455(7215), 960-U42(2003)

[6] G. Giuli, et al., Meteoritics and Planetary Science, 38(8), 1181-1186(2003)

[7] G. Giuli, et al., Meteoritics and Planetary Science, 43(5), 981-986(2008)

[8] R. Arletti, et al., Applied Physics A-Materials Science and Processing, 111(1), 99-108(2013) 